\title{
From loose body to osteochondritis dissecans: a historical account of disease definition
}

\author{
VITTORIO TARABELLA ${ }^{1}$, GIUSEPPE FILARDOํ, BERARDO DI MATTEO ${ }^{1}$, LUCA ANDRIOLO', \\ PATRIZIA TOMBA ${ }^{2}$, ANNA VIGANÒ2, MAURILIO MARCACCI ${ }^{1}$ \\ ${ }_{1}^{1}$ O Orthopaedic and Traumatology Clinic - Biomechanics and Technology Innovation Laboratory; Rizzoli Orthopaedic \\ Institute, Bologna, Italy \\ ${ }^{2}$ Biblioteche Scientifiche IOR, Donazione Putti, Rizzoli Orthopaedic Institute, Bologna, Italy
}

\begin{abstract}
Osteochondritis dissecans $(\mathrm{OCD})$ is a rare yet fascinating disease affecting young, active patients. It remains a 'mysterious disease' whose etiopathology, still unclear, is the subject of ongoing studies aiming improving the knowledge of this condition and, therefore, treatment options, too. Even though the first descriptions of intra-articular loose bodies date back to very ancient times, it is only relatively recently that, thanks to the contribution of some very eminent physicians, it became recognized as a specific orthopaedic condition. The aim of the present manuscript is to trace the main steps in the journey that led to the acknowledgement of OCD as an autonomous clinical entity, and to recall the prominent figures involved.
\end{abstract}

Keywords: osteocondritis dissecans, joint, disease, loose body, etiology.

\section{Introduction}

Osteochondritis dissecans (OCD) is a fascinating clinical entity for several reasons. First of all, it is an uncommon disease, and rarity often stimulates the curiosity and ingenuity of physicians. Second, it is a 'mysterious disease' whose etiopathology, still unclear,

\section{Corresponding Author:}

Luca Andriolo, MD

I Orthopaedic and Traumatology Clinic - Biomechanics

and Technology Innovation Laboratory; Rizzoli

Orthopaedic Institute

Via Di Barbiano 1/10, 40136 Bologna, Italy

E-mail: lucas.andriolo@gmail.com is the subject of ongoing studies aiming at improving the knowledge of this condition and, therefore, treatment options, too. Third, OCD affects different joints with different clinical patterns, and this is another aspect that intrigues clinicians. Last but not least, it is a pathology that has been known for centuries, even before it was given the name currently in use. Historically, knowledge of this condition began in ancient times, with the occasional detection of intraarticular loose bodies within joints - findings that led early physicians to begin enquiring about their origin. The aim of the present manuscript is to trace the main steps in the journey that led to the acknowledgement of OCD as an autonomous clinical entity, and to recall the prominent figures involved. From the first reports of loose intra-articular bodies to the "classical" definition of OCD proposed by König, this historical essay looks at the evolving etiopathogenetic hypotheses that helped to increase knowledge of this disease, and allowed it to be better defined. This analysis is conducted in the awareness that much still needs to be clarified, and that OCD remains an intriguing condition, both for researchers and for physicians dealing with affected patients in their clinical practice.

\section{Loose intra-articular bodies in medical history: a nameless pathology}

The very first documentation of OCD dates back to 1558 when Ambroise Paré (1510-1590), the French "father of surgery", became the first to write about loose bodies removed from the inside of a joint cavity during a surgical intervention. The following extract is taken from his "Stone in Knee (MIII,32)" translated by 
Hamby: "In 1558, I was called by Jean Bourlier, Master Tailor of St. Honoré, to open an aqueous tumor of the knee. In it was found a stone the size of an almond, very white, hard and polished. He recovered and lives at present". (Malgaigne [Joseph-François Malgaigne, friend and editor of Pare's works] says this is the first reported case of removal of a foreign body developing in the knee) (1).

A more accurate description of OCD is dated 1738. Written by Alexander Monro, founder of the Edinburgh Medical School, it is commonly regarded as the first confirmed case analysis of what was, at the time, still a nameless pathology (2).

In Volume Four of his collection of medical essays, the nineteenth chapter is entitled "Part of the Cartilage of the Joint of the Knee separated and ossified". The Author, reporting a "white swelling from a very uncommon cause" discovered by a colleague together with "loose bone" of unknown origin, wrote about a similar experience of his own some years previously: "In the body of a woman aged forty, which I dissected in February 1726, I found, within the ligament of the articulation of the right knee, a bone of the shape and size of a small turkey bean, depending by a ligament balf an inch long from the external side of the tibia. The bone, when cut, had only a thin external firm plate, being composed within of cells which were full of oil. On separating the femur and tibia, I saw the ligament came out from the exterior edge of the cartilage covering the exterior cavity of the tibia; and more internally a part of the cartilage of the tibia, of the same shape with the bone, was wanting".

This instance of two physicians discussing clinical cases and sharing knowledge marks the very beginning of the process by which this condition, initially merely the subject of descriptions of weird anatomical findings (probably due to traumatic causes), assumed the status of a recognizable pathology. Although tibial OCD is uncommon, and from Monro's brief description it is hard to clearly establish the nature of the pathology, his account is traditionally deemed to constitute the first OCD case analysis (3).

A few decades later, in 1759, other observations were provided by Scottish surgeon John Hunter (4): "such detached and moveable cartilages [...] are not peculiar to the joint of the knee; they occasionally occur in other joints of the body; but $[\ldots]$ they are most frequently met with in the knee $[\ldots]$. These substances in their structure are analogous to bone, but in their external appearance bear a greater resemblance to cartilage [...]. The circumstance of their being loose, and having no remains of a visible attachment, made it difficult to form conjectures respecting their formation [...]." These observations have remained valid right up to the present day. In 1817, French physician and inventor of the stethoscope René Laennec wrote of a "joint mouse" not related to trauma, which he suggested to be due to a "proliferation of the cartilage of the periarticular synovial tissue" (5).

Paul Broca, another French physician, before devoting himself to the anatomy of the brain and the founding of anthropology, was a fine researcher of the histology of cartilage and bone. In 1854, in a paper about necrosis of bone and articular cartilage (6), he was the first to suggest that the cause of the loose bodies lay in "spontaneous necrosis" of a part of the articular surface, with a consequent loss of fragments (7).

This was the period when interest in these loose bodies, often called joint mice, was at its peak and in 1855, just a year after Broca's paper, a Leeds surgeon named Thomas Teale published a case report on the topic (8).

"The following case, unfavorable in its result, I am wishful to put on record, on account of its important pathological bearing. John W., Brewer, age 37, of robust frame, but a free liver, became an out-patient of the Leeds Infirmary, in June, 1855. He stated, that a year ago he accidently let a cask, which he was lowering into a cellar, slip against the right knee. Severe pain in the joint followed, and he was unable to work for three weeks; after which time he followed his employment as usual for nearly twelve months, until a few days ago, when he became suddenly lame [...] then, for the first time, felt a flat substance moving about in the knee-joint. [...]

We could distinctly feel the flat substance, about the size of a shilling. It could be readily pushed upwards along the outer side of the joint, then under the extensor tendons, and down the inner side of the joint. It could, again, with equal ease, be made to take a retrograde course, and resume its old place. Very little pain was produced by these forced movements. At times, when the loose body was favorably placed, the patient could walk. pretty well, but on slight changes in its position, extreme pain and lameness would result".

Teale tried, unsuccessfully, to treat the patient with rest and with bandages carefully positioned to keep the joint mouse in a favorable position. He tried many different arrangements of the bandages but none of them succeeded in limiting the movements of the loose body. According to modern standards this could 
be broadly regarded as the first, failed, attempt of conservative treatment.

Under considerable pressure from the patient, Teale agreed to remove the substance from the knee.

"August 1 ${ }^{\text {st }}$, at noon, the patient was brought into the operation room. The knee was but slightly enlarged, and did not show any synovial effusion. The substance being pushed to the outer side of the joint, so as to rest upon the outer condyle, a subcutaneous incision of the synovial membrane was made over it, in the hope that it might then be pushed into the areolar tissue; but this was found to be impracticable, on account of its large size and irregular border.

The skin was then drawn forwards, and divided over the substance, after which it was easily removed. [...] The substance, on being examined, was found to be flattened, circular in form, and irregular or ragged at its border. One of its surfaces had the appearance of cartilage, and was smooth and slightly convex; the other was concave and rough, from a layer of bone.

The operation, unfortunately, was followed by inflammation of the joint and phlegmonous erysipelas of the thigh and buttock, which proved fatal on the sixth day.

The limb was examined after death [...]. At the under surface of the inner condyle the articular cartilage showed a depression, about the eighth of an inch in depth, having rough surface of bone at its base. On comparing this breach in the articular cartilage with the substance which had been removed, they were found to correspond accurately with each other; and, on placing the detached substance in the cavity in the condyle, the continuity of the articular surface was perfectly restored. It is therefore evident that the loose body was a portion of the articular cartilage along with a thin layer of bony substance".

Teale's finding is almost completely ignored by medical historians, even though, preceding König's essay by almost thirty years, it should probably be recognized as the first in-depth analysis of what would later become known as osteochondritis dissecans.

"The explanation, which I venture to offer of this remarkable fact, is that a portion of the articular cartilage, and of the adjoining layer of bone, had been injured by the accident which the patient sustained when moving the cask; and that, by a slow process of exfoliation, extending through a period of about twelve months, the injured part was cast off and became loose in the joint [...]".

Traumatic episodes were most commonly acknowledged to be the cause of these loose bodies: Monro and Teale are just two surgeons whom, by virtue of their important works, we have chosen to mention in this brief excursus, but there were also others who left brief observations on loose bodies.

Loose bodies in the mandibular joint attributed to trauma were reported by Swiss anatomist Baron Albrecht von Haller in 1764 (9), whereas trauma and inflammation were the causes identified by English anatomist George Rainey in 1848, as reported by London surgeon Richard Barwell in his excellent book published in 1881, 'A Treatise on Diseases of the Joints' (in which the whole of chapter IX was devoted to "movable bodies in joints") (10).

Many other theories and observations were proposed in those years (7), but the most important and influential ones prior to König were made in 1870 by Sir James Paget, an English surgeon and pathologist who is considered to be one of the founders of scientific medical pathology.

He offered a different theory about the production of loose bodies in the joints (11). Paget speculated about possible vascular issues, separated and unrelated to injuries or inflammation, referring to a phenomenon that he called "quiet necrosis". In his paper he reported two different cases, which he assessed using a more systematic and targeted approach than Teale had adopted, a clear sign of the now increased interest in this condition.

"A girl was admitted under my care with a painful periosteal swelling on the middle and outer part of the femur. After various useless treatments, I cut down to the bone - cutting through perfectly bealthy skin, fat, fascia, and muscles - and found, between the thickened periosteum and a small hollow in the wall of the femur, a loose piece of bone about an inch long and a quarter of an inch wide. This was a sequestrum, separated from the wall of the femur, without suppuration or any other severer inflammation that had produced the thickening of the periosteum.

The origin of this quiet necrosis and exfoliation was a babit the girl had of breaking thick pieces of wood across her thigh. The case [...] is enough to prove that necrosis of bone may take place, and the dead bone may be exfoliated, without the usually attendant suppuration and other signs of destructive inflammation". The second case was that of a sportsman aged sixteen, very active, who had never suffered any particular accidents or injuries to the joint: "He had had, for nearly a year, all the usual signs of a loose body in the right knee; and could usually, without difficulty, by shaking and pressure about the joint, bring one into the upper pouch of synovial membrane on either side". 


\section{Joints}

$\jmath$

Paget proceeded surgically, removing a piece of cartilage from the young man's knee.

"I extracted the loose body through a free incision into the joint, and the wound healed without trouble.

This body looked exactly like a piece of the articular cartilage of one of the condyles of the femur. It was irregularly oval in outline, about an inch long, balf an inch wide, and a line in thickness. On one surface it was convex and smooth, on the other concave and rough; and on this surface was a small prominent piece of bone, as if, with the cartilage, a piece of the articular surface of the femur had separated. The borders of the loose body were smoothly rounded off".

What follows is an accurate and detailed description of the loose body, the first of its kind.

"In agreement with this general likeness to a piece of articular cartilage from a condyle was the microscopic structure of this loose body. In sections through its thickness was found a nearly homogeneous basis-substance, with cartilage corpuscles which, in arrangement and all their other characters, were exactly like those of articular cartilage. At and near the concave surface they were elongated, with their longer diameters at right angles with the surface and grouped in nearly parallel vertical lines. Nearer to the convex surface they were round or oval, fewer, and irregularly placed; and just beneath this surface they were numerous, this, compressed and flattened parallel with the surface, which was dense and darkly defined, exactly like the synovial surface of an articular cartilage".

Paget was certain about the origin of the loose body: he was in no doubt that it came from the cartilage and bone of the joint itself.

"But how can such pieces of articular cartilage be detached from living bone? [...] They cannot be chipped off; no force can do this; and they are not like the fragments of condyle which are sometimes, in violent fractures, broken off the femur and left loose of pendulous in the joint".

The theory that Paget went on to elaborate was that of a combination of injury, not necessarily extremely violent, and the aforementioned quiet necrosis: "These loose bodies are sequestra, exfoliated after necrosis of injured portions of cartilage, exfoliated without acute inflammation, just as the piece of bone was, or as a tooth after a blow may be slowly detached from its alveolus and cast out.

And certainly, among all the tissues, there is none in which it might be more expected than in articular cartilage that the separation of dead pieces should take place without acute inflammation; for, its substance being without blood vessels and not easily permeable, products of its morbid changes cannot be easily diffused".
In short, according to the author, loose bodies were exfoliated after necrosis due to mild trauma, often in young and healthy people, and always weeks after the possible injury.

The injury, "when a particular injury can be assigned", does not break off the loose body, it simply causes the beginning of the necrosis process or, maybe, just accelerates it.

Although Paget was very well informed about the experiences and works of others, such as Broca, who belonged to the vascular cause school of thought, he realized that most physicians would accept his more straightforward explanation of trauma as the cause of loose bodies.

"For these cases, I believe the explanation I have offered is the true one. Just as a blow on a bone or on a tooth may induce necrosis and exfoliation without any signs of destructive inflammation, so may it be with articular cartilage: and the characteristics of these cases will be that, after injury to a previously bealthy joint, a loose body is found in it, having the shape and general aspect and texture of a piece of articular cartilage, with or without some portion of subjacent bone, and with its cartilage-corpuscles arranged after the manner of those of articular cartilage".

Paget had the ingenuity to combine previous studies, by different physicians, with his direct experience and analysis, and was thus able to reach a deep understanding of the pathology and its working. The only thing Paget did not do was giving it a name.

\section{Osteochondritis dissecans}

In the wake of these developments, physicians soon began to identify (and also look for) cases of loose bodies in joints but, as they say, there is power in names and, in 1887, Franz König (1832-1910) was the one who finally came up with a name for the pathology responsible for the presence of loose bodies in joints (12), and also produced the period's most comprehensive study of the condition.

The English translation of the paper was recently digitalized and made readily accessible to all (12). "The history of loose osteochondral bodies, the free bodies, in buman joints, the joint mice, as they were called by our predecessors in a naive way due to their rapid movements is in some way reminiscent of a mouse scurrying about inside the joint sacs. Since 
antiseptic surgery we can not only remove the loose bodies but also view the joint itself and make observations on the in vivo factors for the formation of the bodies. Also in relation to the presence of these bodies in the different joints, our knowledge has expanded since that time, and if the surgeon earlier in the discussion of "joint mouse" almost invariably thought of the knee joint, we now know these bodies occur in other joints as well". After this brief introduction, the paper immediately tackles three different cases personally treated by König, and on the basis of this case analysis the author then proposes his theory about the formation of loose bodies in joints.

"In all 3 cases it was youthful individuals who had the disorder (16, 20 and 12 years at the first onset of symptoms). [...] We want to highlight that none of the patients had any general joint diseases, especially none was affected by arthritis deformans, and that the joint for all three individuals other than the locale capsule thickening as consequence of the stimulus of the foreign body and that the defect in the first patient, to which we shall return later, was associated with no signs of general disease such as arthritis deformans. In common all three patients had similar clinical symptoms. Sudden pain occurred in the affected joint frequently with swelling, and then with disappearance of the initially severe symptoms which was associated with painful restricted mobility, there were function restrictions for shorter or longer periods. This functional disturbance was regularly accompanied by limited extension of the joint".

König's paper had the intrinsic merit of taking the examination of loose bodies to the next step: it did not just observe the condition, or confine itself to presenting a strange anatomical case. Indeed, as well as wishing to share his theory, König also wanted to become the guide and point of reference for the treatment of it. His paper therefore provided directions on how to operate, what to avoid, and the best way to treat the wound after surgery. In fact, most of the paper is made up of analysis of symptoms, and suggestions for diagnosis and treatment: "[...] we can soon put together the symptoms to suggest the probability or for reliably diagnosing moving bodies in the elbow joint: when repeated attacks of sudden pain in the relevant joint occur with symptoms of synovitis, when moving the joint is remarkably painful and if after the disappearance of the worst symptoms there is a restriction on the extension of the joint for a longer period, it is very likely that it is a joint mouse. [...] The surgical removal of the body from the elbow joint is extremely rewarding" (Fig. 1).

Moreover, König wanted somehow to put an end to the wild speculations, and summarize the state of knowledge on what was still a disorder without a name. "The doctrine of the origin of the free body is definitely still not completely closed and especially the questions, can a free body in a joint form by an injury and how often are joint bodies of traumatic origin, are certainly not answered by either the pathological anatomist or surgeon. If we now want to deny on the one hand that there are traumatic loose bodies, for example, that the radial head can break off in whole or in part, and immediately in the joint cause the symptoms of a free body, we believe on the other hand that the majority of cases of in which the joint mice have been described following trauma, cannot be considered in the strict sense resulting from a broken-off body".

This is what was known at the time, and König put a symbolic hat on it by coining the name osteochondritis dissecans (OCD).

The paper concluded with descriptions of five more cases and a summary of the agreed knowledge on OCD, and thus marked the starting point from which to undertake future research:

"Let us summarize the conclusions of our view of the importance of trauma in the development of mobile joint bodies; we will formulate the same as follows.

1. The occurrence of immediate loose bodies brought about by an injury to the articular surface is relatively rare in bealthy joints and conceivable only as a result of severe trauma.

2. From such violent actions loose pieces of the articular surface can occur by avulsion with ligaments, or even entire sections of a joint surface, such as the radius head, the femoral head, can be prevented by a levering effect dissipating the violence or also by the same violence inducing a lateral piece. However, it is absolutely inconceivable that flat pieces of the surface of an articular

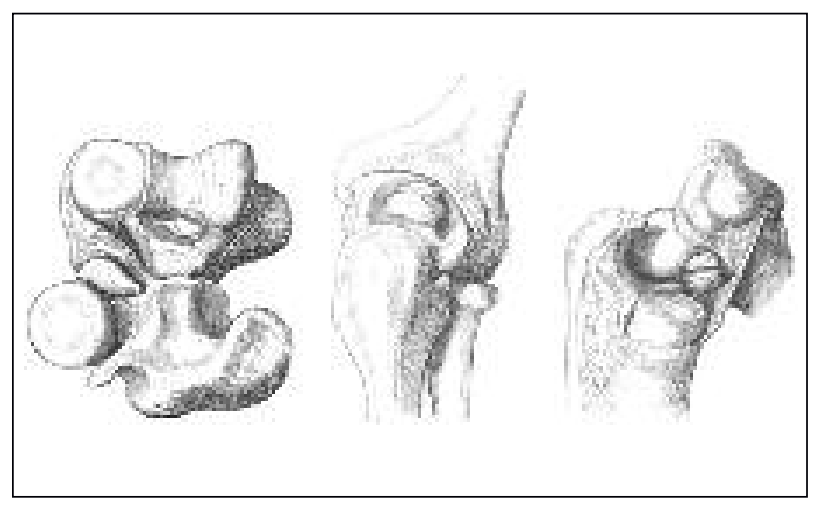

Fig. 1. Original drawings from the paper by F. König, showing multiple OCD inside the elbow joint. 


\section{oints}

surface, as we have described in the elbow joint of and the knee, are immediately detached by a traumatic event without any serious injury to the joint.

3. It is quite conceivable that such pieces are so subject to injury, that the same necrosis with subsequent dissecting inflammation leads to their separation.

4. There is a spontaneous osteochondritis dissecans, which without any other considerable damage to the joint brings about detached pieces of the articular surface. A great part of remote traumatic events associated with loose bodies must be considered as having occurred in this way.

5. The etiology of the proposed pathological processes is still unknown".

\section{Conclusions}

Almost three hundred and fifty years passed between the first documented view of a joint "mouse" and the day that mouse was given a name.

From Paré to König, this journey involved, over the decades, a multitude of scientists and the patients they treated: each played a part in this process to progress, a process that continued after König and is still going on today.

Some aspects have been questioned, and even the term osteochondritis dissecans is now regarded by many as euphonic and 'catchy', but rather incorrect, since inflammation is not a peculiar feature of the primary lesions. But, as this brief historical note shows, the never-ending flow of medical progress goes on, regardless of names and definitions, towards the discovery of the causes of diseases and their treatment. The work of these forerunners in the definition of OCD laid the foundations for ongoing research aimed at improving the treatment of this disease in everyday clinical practice.

\section{Acknowledgements}

The authors would like to thank St Bartholomew's Hospital Archives \& Museum of London, Liliana Draghetti (Donazione Putti, Biblioteche Scientifiche, Rizzoli Orthopaedic Institute), Keith Smith, Davide Puppini, Linda Accorsi for their help.

The present paper was supported by the following funds granted to Rizzoli Orthopaedic Institute: “5\%o (5 per mille), anno 2012".

\section{References}

1. Paré A. The case reports and autopsy records of Ambroise Paré. 1560. In: Hamby WB (Ed.) Translated from J. P. Malgaigne's Oeuvres completes d'Ambroise Paré Paris, 1840. Charles C Thomas, Springfield: p.113.

2. Monro A. Medical Essays and Observations. Vol 4. Edinburgh Society for the Improvement of Medical Knowledge, Edinburgh. 1738:305-306.

3. Nagura S. Problem of so-called osteochondritis of the femur head. Z Orthop Ihre Grenzgeb. 1959;91:224-239.

4. Hunter J. Some observations on the loose cartilages found in joints, and most commonly met with in that of the knee. In: Hunter J (Ed.) The Complete Works of John Hunter. Vol. 3. Haswell, Barrington and Haswell, Philadelphia. 1841:583-588.

5. Brand R. 50 years ago in CORR: the so-called osteochondritis dissecans of König Shigeo Nagura, MD CORR 1961;18:100122. Clin Orthop Relat Res. 2011;469:2975-2976.

6. Broca P. Sur la nécrose des cartilages articulaires. Aerzte, Paris. 1854;38.

7. Cugat R, Garcia M, Cusco X, et al. Osteochondritis dissecans: a historical review and its treatment with cannulated screws. Arthroscopy. 1993;9:675-684.

8. Teale TP. Case of detached piece of articular cartilage existing as a loose substance in the knee-joint. Med Chir Trans. 1856;39:31-33.

9. Shah SB, Ramanojam S, Gadre PK, et al. Synovial chondromatosis of temporomandibular joint: journey through 25 decades and a case report. J Oral Maxillofac Surg. 2011;69: 2795-2814.

10. Barwell R. A Treatise on Diseases of the Joints. Wood, New York. 1881:180-182.

11. Paget J. On the production of some of the loose bodies and joints. St Bartholomew's Hosp Rep. 1870;6:1-4.

12. König F. The classic: On loose bodies in the joint. 1887. Clin Orthop Relat Res. 2013;471:1107-1115. 